\title{
A review of geriatric injuries at a major trauma centre in South Africa
}

\author{
J-P Da Costa; ${ }^{1}$ J Laing, ${ }^{2}$ BSc (Dietetics), MB ChB; V Y Kong, ${ }^{2,3}$ MB ChB, ChM, MSc, PhD, MRCS (Ed); J L Bruce, ${ }^{2}$ MB ChB, FCS (SA); \\ G L Laing, ${ }^{2}$ MB ChB, MSc, FCS (SA), Cert Trauma Surgery (SA), PhD; D L Clarke, ${ }^{2,3}$ MB ChB, MPhil, MBA, PhD, FCS (SA) \\ ${ }^{1}$ Final-year medical student, Faculty of Health Sciences, University of the Witwatersrand, Johannesburg, South Africa \\ ${ }^{2}$ Department of Surgery, Nelson R Mandela School of Medicine, College of Health Sciences, University of KwaZulu-Natal, Durban, South Africa \\ ${ }^{3}$ Department of Surgery, Faculty of Health Sciences, University of the Witwatersrand, Johannesburg, South Africa
}

Corresponding author: V Y Kong (victorywkong@yahoo.com)

\begin{abstract}
Background. Trauma in South Africa (SA) has been referred to as a malignant epidemic, but the impact of trauma on the elderly has tended to be overlooked.

Objectives. To address this deficit by focusing on trauma victims aged $\geq 65$ years.

Methods. All patients aged $\geq 65$ years who were admitted to Grey's Hospital, Pietermaritzburg, SA, following trauma between December 2012 and January 2019 were reviewed.

Results. Over the 6-year study period, a total of 281 patients aged $\geq 65$ years were admitted to Grey's Hospital following trauma. There were 150 males (53.4\%) and 97 females (34.5\%). The sex of 34 patients was unknown. The average age was 72 years (range 65 - 97). There were 226 cases of blunt trauma, 42 cases of penetrating trauma (including two incidents of impalement following blunt trauma) and 15 cases of other types of trauma. The most common causes of blunt trauma were accidental falls $(n=76)$, motor vehicle accidents $(n=46)$, pedestrian vehicle accidents $(n=32)$ and falls from a height $(n=23)$. Gunshot wounds $(n=22)$ and knife wounds $(n=14)$ were the most common forms of penetrating trauma. Other trauma mainly comprised dog bites $(n=6)$ and snakebites $(n=6)$. There were 72 incidents of assault ( $25.6 \%$ of total cases). The majority of assaults were committed by a single perpetrator, and the perpetrator was frequently known to the victim. There were no significant differences in the proportions of penetrating, blunt and other trauma injuries between males and females. A total of 44 patients (15.7\%) required surgical intervention, and 41 patients (14.6\%) experienced complications during their hospitalisation. Respiratory, renal and cardiac complications were most frequent, and 5 patients had a cardiac arrest. Seven experienced acute kidney injury. Seventeen patients $(6.0 \%)$ required intensive care unit admission and $5(1.8 \%)$ required ventilation. Patients stayed in hospital for an average of 2.96 days (range 0 - 39). Of the patients, $241(85.8 \%)$ survived, $32(11.4 \%)$ died and $8(2.9 \%)$ had an unknown outcome.

Conclusions. Geriatric trauma in SA is relatively rare, but will increase as the population ages. There is a high incidence of assault as a mechanism, highlighting the fact that elderly people are a vulnerable group. Managing these patients is challenging and is associated with significant morbidity and mortality.
\end{abstract}

S Afr Med J 2020;110(1):44-48. https://doi.org/10.7196/SAMJ.2020.v110i1.14100

Trauma in South Africa (SA) has aptly been referred to as a 'malignant epidemic. ${ }^{[1,2]}$ It places a massive burden on society as a whole as well as on the healthcare system. SA, like many low- to middle-income countries (LMICs), is considered to be a 'young' country with a population pyramid weighted heavily towards people $<40$ years of age. ${ }^{[3,4]}$ Focus has been placed on improving outcomes for children and young people. Maternal and child health and the antiretroviral programme have dominated health policy and have absorbed much health funding. ${ }^{[3]}$ This situation is gradually changing. As infectious disease is brought under control, there is growing awareness of the burden of non-communicable disease. ${ }^{[1,5]}$ Surgical diseases contribute significantly to the overall burden of non-communicable disease, and trauma is a major surgical disease. Trauma is traditionally thought of as affecting young people. While this is true for the most part, certain vulnerable groups deserve special attention. Much has been published on the burden of trauma in SA over the past half century. Despite this extensive literature, however, children, women and the elderly are relatively under-represented and under-researched.

\section{Objectives}

The impact of trauma on the elderly has tended to be overlooked, and the objective of this study was to address this deficit by focusing on trauma victims aged $\geq 65$ years.

\section{Methods}

\section{Clinical setting}

The Pietermaritzburg Metropolitan Trauma Service (PMTS) is based at Grey's Hospital in the city of Pietermaritzburg, SA. It provides trauma care to the city of Pietermaritzburg with its population of one million people and tertiary trauma care to the predominantly rural western third of KwaZulu-Natal (KZN) Province. ${ }^{[6]}$ This is a large geographical area served by 19 district hospitals and 2 regional hospitals, with a population of 2 million people. ${ }^{[6]}$ The PMTS is one of the largest academic trauma centres in western KZN. A digital database known as the Hybrid Electronic Medical Registry (HEMR) captures data on all patients admitted to the trauma centre. ${ }^{[6]}$ Ethics approval for maintenance of the registry and for this study was 
formally granted by the Biomedical Research Ethics Committee of the University of KwaZulu-Natal (ref. nos BCA 207/09 and BCA 221/13).

\section{The study}

All patients aged $\geq 65$ years who were admitted to the PMTS following trauma between December 2012 and January 2019 were identified from the HEMR. ${ }^{[6]}$ Basic demographic data regarding the mechanism and severity of the injuries were reviewed. Clinical outcomes were assessed.

\section{Results}

Over the 6-year period, a total of 8722 trauma patients were admitted to Grey's Hospital. ${ }^{[6]}$ The average age was 29.7 years. ${ }^{[6]}$ A total of 5027 patients sustained blunt trauma (57.6\%) and 3334 (38.5\%) sustained penetrating trauma. ${ }^{[6]}$ The mortality rate for all trauma admissions was $4.5 \%(n=396) .{ }^{[6]}$ Of all the trauma admissions, $281(3.2 \%)$ patients were aged $\geq 65$ years. In this group there were 150 males $(53.4 \%)$ and 97 females (34.5\%). The sex of 34 patients was unknown. The average age was 72 years (range 65 - 97). There were 226 instances of blunt trauma, 42 cases of penetrating trauma (including 2 cases of impalement following blunt trauma) and 15 cases of other types of trauma (Fig. 1). The most common causes of blunt trauma were accidental falls $(n=76)$, motor vehicle accidents $(n=46)$, pedestrian vehicle accidents $(n=32)$ and falls from a height $(n=23)$. The most common forms of penetrating trauma were gunshot wounds $(n=22)$ and knife wounds $(n=14)$. Other trauma mainly comprised dog $(n=6)$ and snake $(n=6)$ bites. Table 1 breaks down the mechanisms of trauma in the study cohort.

There were 72 incidents of assault (25.6\%). The majority of assaults were committed by a single perpetrator. The perpetrator was frequently known to the victim. Table 2 summarises the data for the victims of assault.

There were no significant differences in the proportions of penetrating, blunt and other trauma injuries between males and females.

\section{Physiological parameters}

On admission, patients had an average shock index of 0.64 (range 0.27 - 1.29) and an average Revised Trauma Score of 7.38 (range 1.76 7.8408). Table 3 summarises the admission physiological parameters for the cohort.

\section{Imaging}

The most commonly used modes of radiological investigation were computed tomography scans $(n=210)$ and chest radiographs $(n=128)$. The least commonly used were FAST (focused assessment with sonography in trauma) $(n=1)$, magnetic resonance imaging $(n=2)$

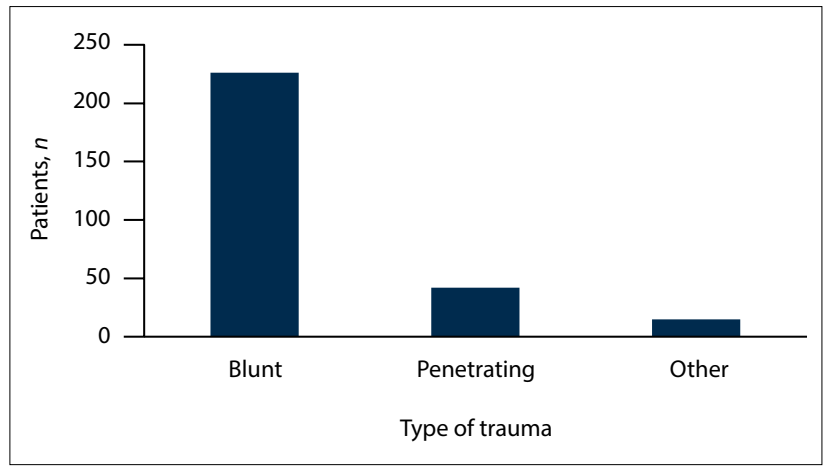

Fig. 1. Types of trauma ( $\mathrm{N}=281$ patients, 283 mechanisms of injury). and formal ultrasound ( $n=7)$. Fig. 2 shows the imaging modalities used in this cohort.

\section{Injuries}

The most frequently injured body regions were the head $(n=164)$ and the thorax $(n=67)$. The majority of patients with head injuries presented with subarachnoid haemorrhage $(n=35)$ or subdural haemorrhage $(n=35)$; lacerations, abrasions and contusions, together with closed skull vault fractures, were the next most common injuries to the head and face. The most prevalent neck injury was fracture of the C1 - C7 vertebrae. A unilateral rib fracture $(n=24)$ was the most common thorax injury. The most frequent pelvic injury was a closed pelvic fracture $(n=11)$. The most common lower limb injuries were fractures of the tibia $(n=17)$, fibula $(n=13)$ and femur $(n=8)$, and puncture wounds $(n=12)$. Fig. 3 provides a breakdown of injury according to body region.

\begin{tabular}{ll} 
Table 1. Mechanisms of injury $\left(\mathbf{N}=\mathbf{2 8 1} \mathbf{1}^{\star}\right)$ \\
\hline & $\boldsymbol{n}$ (\% of injuries \\
sustained by this \\
mechanism in \\
respective categories) \\
\hline Blunt trauma & 226 \\
Accidental fall & $76(33.6)$ \\
Motor vehicle accident & $46(20.3)$ \\
Pedestrian vehicle accident & $32(14.2)$ \\
Fall from height & $23(10.2)$ \\
Assault, unknown item & $15(6.7)$ \\
Fall from moving vehicle & $7(3.1)$ \\
Unknown & $4(1.8)$ \\
Assault, wooden rod & $4(1.8)$ \\
Motorbike accident & $3(1.3)$ \\
Assault, pistol & $2(0.9)$ \\
Assault, rock & $2(0.9)$ \\
Assault, strangulation & $2(0.9)$ \\
Assault, axe & $1(0.4)$ \\
Assault, brick & $1(0.4)$ \\
Assault, hammer & $1(0.4)$ \\
Assault, metal rod & $1(0.4)$ \\
Assault, rake & $1(0.4)$ \\
Assault, rope & $1(0.4)$ \\
Kick to head (goat/cow) & $1(0.4)$ \\
Person falling onto patient & $1(0.4)$ \\
Seizure & $1(0.4)$ \\
Trampled by cow & $1(0.4)$ \\
Penetrating trauma & 42 \\
Gunshot & $22(52.4)$ \\
Knife & $14(33.3)$ \\
Impalement & $2(4.8)$ \\
Axe & $1(2.4)$ \\
Buffalos, as 2 patients sustained two mechanisms of injury. \\
Iron rod & $1(6.7)$ \\
Rake & $1(2.4)$ \\
Other types of trauma & $1(2.4)$ \\
Dog bite & 15 \\
Snakebite & $6(40.0)$ \\
Bite, unspecified & $6(40.0)$ \\
Hospital transport & $1(6.7)$ \\
& $1(6.7)$ \\
& \\
& \\
&
\end{tabular}




\begin{tabular}{ll} 
Table 2. Details of cases of assault & \\
\hline Total cases of assault, $n$ (\% of all patients) & $72 / 281(25.6)$ \\
Familiarity with perpetrator, $n$ (\%) & $11(15.3)$ \\
Known & $11(15.3)$ \\
Unknown & $50(69.4)$ \\
$\quad$ Not specified & $18(25.0)$ \\
Number of perpetrators, $n(\%)$ & $5(6.9)$ \\
One & $49(68.1)$ \\
More than one & \\
Not specified & \\
Relation of perpetrator to victim & \\
(where specified), $n$ & 3 \\
Intruders & 3 \\
Grandson & 2 \\
Son & 1 \\
Daughter in law & 1 \\
Domestic worker & 1 \\
Family member (unspecified) & 1 \\
Wife &
\end{tabular}

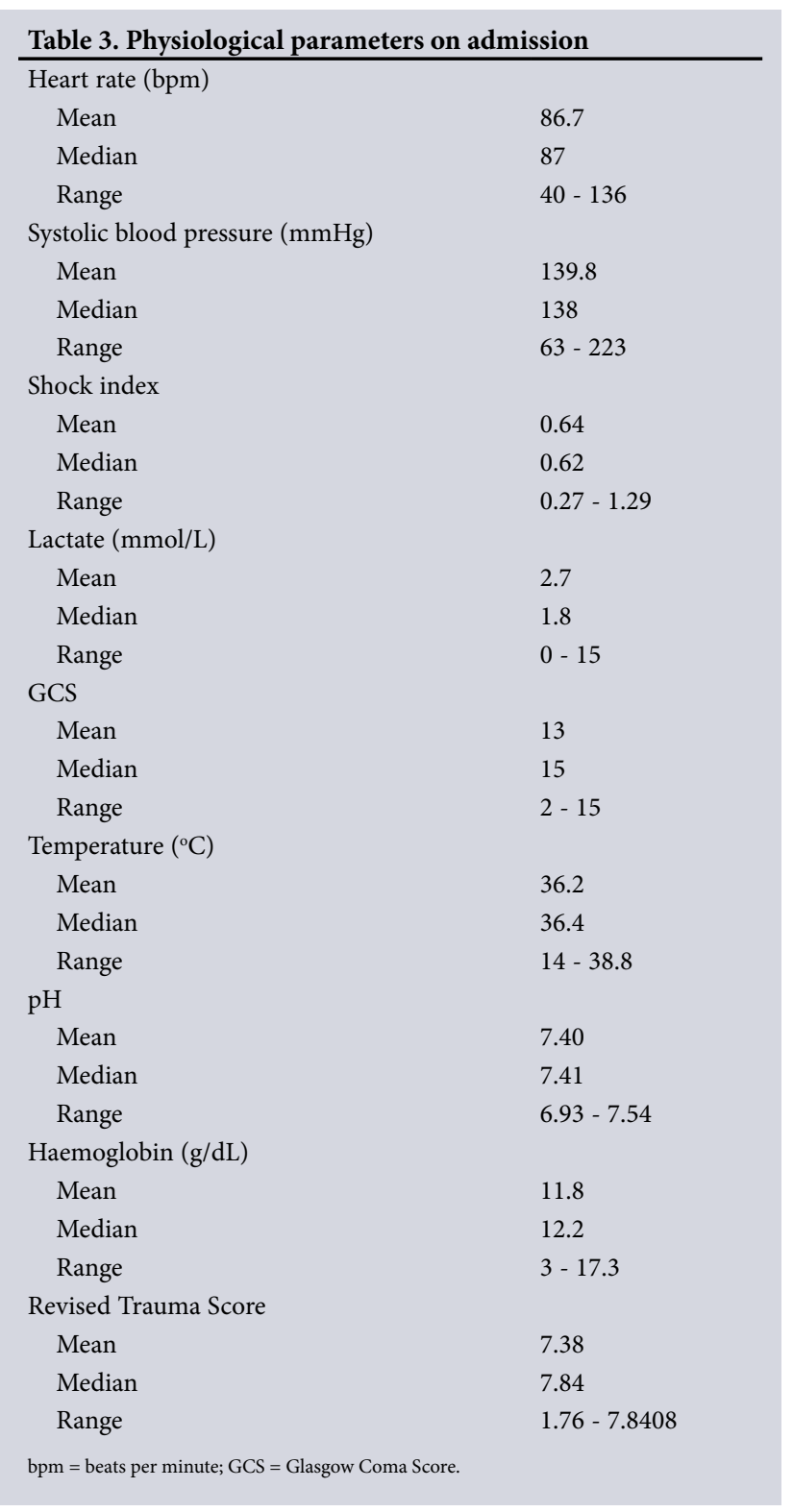

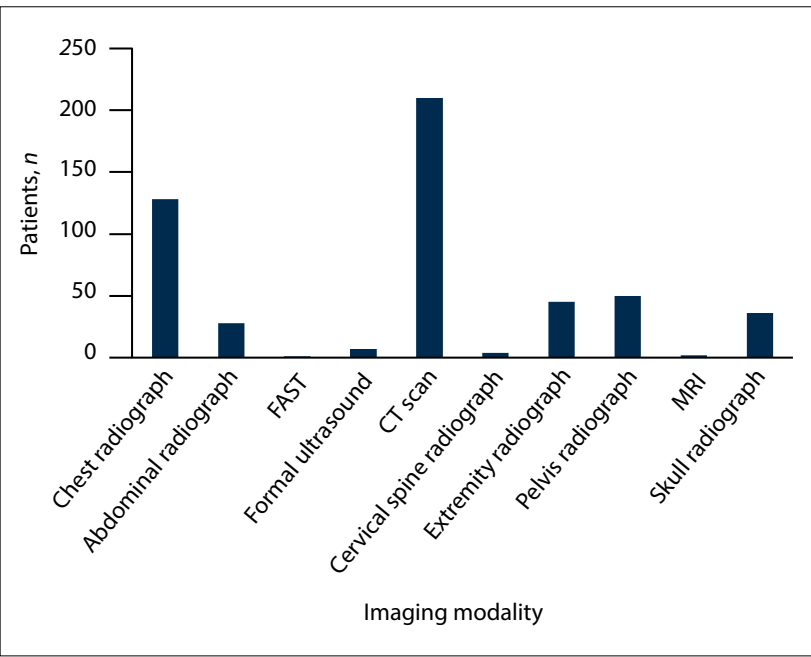

Fig. 2. Radiological investigations $(\mathrm{N}=281$ patients $) . \quad(F A S T=$ focused assessment with sonography in trauma; $C T=$ computed tomography; $M R I=$ magnetic resonance imaging.)

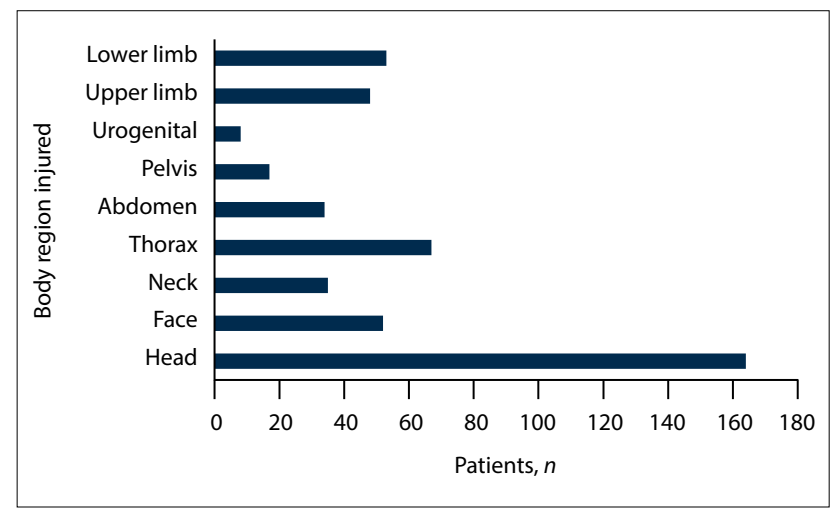

Fig. 3. Body regions injured ( $\mathrm{N}=281$ patients).

\section{Outcomes}

A total of 44 patients (15.7\%) required surgical intervention. The most common procedures were wound debridement $(n=15)$, laparotomy $(n=10)$ and tracheostomy $(n=8)$. Table 4 summarises the operative procedures. A total of 41 patients (14.6\%) experienced complications. Respiratory, renal and cardiac complications were most common, and 5 patients had a cardiac arrest. Seven patients experienced acute kidney injury. Seventeen patients (6.0\%) required intensive care unit (ICU) admission, and 5 (1.8\%) required ventilation. Patients stayed in hospital for an average of 2.96 days (range 0 - 39). Of the patients, $241(85.8 \%)$ survived, $32(11.4 \%)$ died and $8(2.9 \%)$ had an unknown outcome. The mortality rate for all trauma admissions was $4.5 \%$.

There was no obvious relationship between patient age and outcome. Penetrating injury (10.0\%) and blunt trauma (10.5\%) had similar mortality rates, and these rates were approximately half the rate for other trauma (20.0\%). Gunshot injuries (18.2\%) and falls from a height $(14.3 \%)$ were associated with the highest mortality rates, while knife injuries (7.1\%) and pedestrian vehicle accidents (6.3\%) had the lowest mortality rates. Males (11.7\%) had a slightly higher mortality rate than females $(8.5 \%)$. Of the patients who underwent an operation, $13.9 \%$ died, of those who were admitted to the ICU, $29.7 \%$ died, and of those who required mechanical ventilation, $28.0 \%$ died.

There was an inversely proportional relationship between the Revised Trauma Score and mortality (Fig. 4), an approximately proportional relationship between the shock index and mortality 


\begin{tabular}{ll} 
Table 4. Operative procedures & \\
\hline Patients requiring surgical intervention, & $44 / 281$ (15.7) \\
$n$ (\% of all patients) & \\
Procedures performed, $n$ & \\
Wound debridement & 15 \\
Laparotomy & 10 \\
Tracheostomy & 8 \\
Internal fixation & 4 \\
Split-skin graft & 4 \\
Fasciotomy & 3 \\
Percutaneous endoscopic gastrostomy & 3 \\
Amputation & 2 \\
Exploratory laparotomy & 2 \\
Thoracoscopy & 2 \\
Colostomy & 1 \\
Craniectomy & 1 \\
Craniotomy & 1 \\
End ileostomy & 1 \\
External fixation & 1 \\
Femoral exploration & 1 \\
Groin exploration & 1 \\
Hemicolectomy & 1 \\
K-wire insertion & 1 \\
Laryngeal repair & 1 \\
Laryngoscopy & 1 \\
Laparoscopy & 1 \\
Ligation of profunda femoris artery & 1 \\
Neck exploration & 1 \\
Plate mandible & 1 \\
Removal of subcutaneous bullet & 1 \\
Repair of facial injuries & 1 \\
Rib fracture plate & 1 \\
Transverse colon resection & 1 \\
Upper endoscopy & 1 \\
Wiring of mandible & 1 \\
Vascular repair & \\
& 1 \\
& 1 \\
& 1 \\
& 1 \\
\hline
\end{tabular}

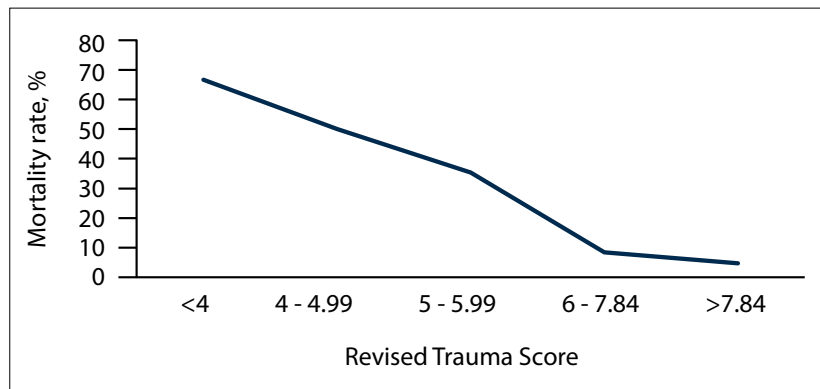

Fig. 4. Relationship between the Revised Trauma Score and mortality.

(Fig. 5), and a well-defined proportional relationship between the Injury Severity Score and mortality (Fig. 6).

\section{Discussion}

The face of trauma in the developed world is changing as the population ages. Data from the UK Trauma Audit Research Network reveal that the mean age of a patient suffering major trauma increased from 36.1 years in 1990 to 53.8 years 23 years later, and the proportion of major trauma patients aged $>75$ years increased dramatically from

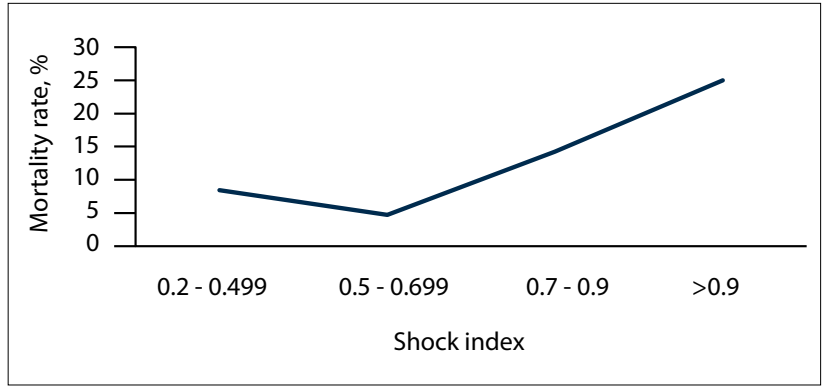

Fig. 5. Relationship between the shock index and mortality.

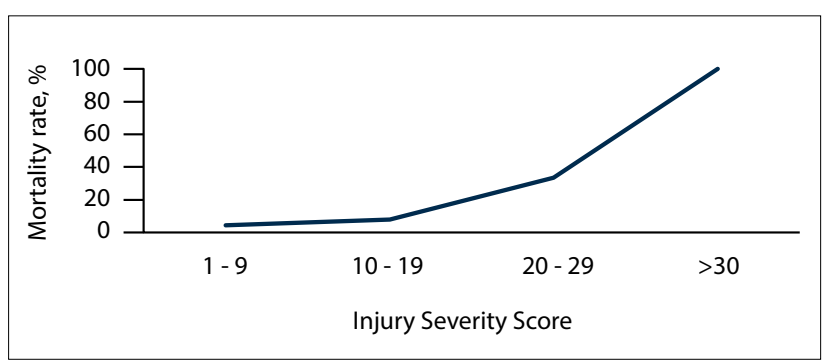

Fig. 6. Relationship between the Injury Severity Score and mortality.

$8.1 \%$ to $26.9 \%$ during the same period. ${ }^{[1,7]}$ The situation in LMICs is different, and SA, in common with many developing countries, has a young population, with the burden of trauma still tending mainly to affect young males. ${ }^{[3]}$ However, trauma in SA is ubiquitous and therefore directly impacts on all sectors of the population. Some of these sectors are particularly vulnerable to trauma, yet historically have tended to be under-researched and overlooked in the academic literature. One such vulnerable group is individuals aged $\geq 65$ years. Trauma in this age group presents unique challenges in terms of management and aetiology. The Advanced Trauma Life Support (ATLS) dedicates an entire chapter to trauma in the elderly and stresses that management of trauma in these patients is complicated by anatomical and physiological changes as well as associated comorbidities. Pre-existing cardiac disease or medications may complicate the resuscitation process. Relatively minor mechanisms such as falls and slips may result in significant injuries. In addition, the elderly are vulnerable to neglect and even elder abuse. ${ }^{[8,9]}$ All these factors complicate the management of elderly trauma patients and make it different from the management of younger patients. Their outcome appears to be worse than that for younger patients, with a mortality rate almost two and a half times as high. ${ }^{[6]}$ As SA develops, the proportion of elderly patients will increase. The continued lack of resources for managing the huge burden of trauma in the public healthcare system poses specific difficulties for these patients. In particular, geriatric rehabilitation and nursing home facilities are almost non-existent in the public sector, and the elderly often occupy acute-care hospital beds for many days.

In stark contrast to the developed world, which is grappling with the challenges of an ever-ageing population, geriatric trauma is relatively rare in our setting, with only 281 patients being admitted over the 6-year study period, which equates to $<50$ cases per year. ${ }^{[7]}$ This low incidence rate is consistent with the overall demographics of the country, which has a mean life expectancy of 50 years. In keeping with the international experience, the most common cause of injury in the present study was blunt trauma. However, this is in complete contrast to the spectrum of trauma in SA as a whole, where penetrating trauma is much more prominent. The high prevalence of penetrating trauma in the elderly and the assault rate of $25.3 \%$ 
are almost unheard of in the developing world, reflect the high rate of interpersonal trauma in SA as a whole, and demonstrate that the elderly are not exempt from being victims of crime and violence. Of note, in cases where the relationship of the perpetrator to the victim could be ascertained, half of the assailants were living with the victim. The elderly are particularly vulnerable members of society and are at risk for assault and abuse from people with whom they live. ${ }^{[9,10]}$ Elder trauma and abuse is a growing concern around the world, and SA, with its high overall incidence of trauma, is no exception.

Accidental falls accounted for $33.6 \%$ of blunt trauma and road traffic collisions for a further $35.8 \%$. Accidental falls are an everpresent threat in the elderly, and their decreased agility and poor eyesight, as well as comorbid illnesses, combine to place them at risk from these relatively minor mechanisms of injury. The elderly are particularly at risk for road traffic-related injuries, as their reflexes tend to be slow and they cannot move out of harm's way as rapidly as a younger person. The tremendous burden of road traffic-related trauma in SA is a neglected epidemic in its own right and affects children, women and the elderly as well as young men. ${ }^{[1]}$ There is a pressing need for a co-ordinated and multifaceted response to this epidemic, involving education of the general public, engineering to separate pedestrians from traffic, and improved enforcement of traffic laws by the authorities.

\section{Conclusions}

Geriatric trauma in SA is relatively rare, but will increase as the population ages. There is a high incidence of assault as a mechanism, highlighting the fact that elderly people are a particularly vulnerable group. Managing these patients is challenging and is associated with significant morbidity and mortality rates.

\section{Declaration. None.}

\section{Acknowledgements. None.}

Author contributions. J-PDC: data collection and analysis, literature review, manuscript drafting; JL: data management and capture; VYK concept and manuscript drafting; JLB: data management and capture; GLL: data capture and database design; DLC: senior author, proofreading, corrections and overseeing the writing of the manuscript.

Funding. None.

Conflicts of interest. None.

1. Muckart D. Trauma - the malignant epidemic. S Afr Med J 1991;79(2):93-95.

2. Clarke D, Thomson S, Madiba T, et al. Selective conservatism in trauma management: A South African contribution. World J Surg 2005;29(8):962-965. https://doi.org/10.1007//500268-005-0131-9

3. Hardcastle T, Oosthuizen G, Clarke D, et al. Trauma, a preventable burden of disease in South Africa: Review of the evidence, with a focus on KwaZulu-Natal. In: South African Health Review. Durban Health Systems Trust, 2016:179-190.

4. Lutge E, Moodley N, Tefara A, et al. A hospital based surveillance system to assess the burden of trauma in KwaZulu-Natal Province, South Africa. Injury 2016;47(1):135-140. https://doi.org/10.1016/j. injury.2015.08.020

5. Clarke D. 'The malignant epidemic' remains the neglected stepchild. S Afr J Surg 2017;55(4):2.

6. Donovan M, Kong V, Bruce J, et al. The Hybrid Electronic Medical Registry allows benchmarking of quality of trauma care: A five-year temporal overview of the trauma burden at a major trauma centre in South Africa. World J Surg 2019;43(4):1014-1021. https://doi.org/10.1007/s00268-018-04880-1

7. Fisher J, Bates C, Banerjee J. The growing challenge of major trauma in older people: A role for comprehensive geriatric assessment? Age Ageing 2017;46(5):709-712. https://doi.org/10.1093/ageing/ afxo35

8. Altintas H, Aslan G. Prevalence of elder abuse among community-dwelling older adults in Turkey and its associated factors. Psychogeriatrics 2019 (epub 28 November 2019). https://doi.org/10.1111/ psyg. 12446

9. Burnes D, Pillemer K, Caccamise PL, et al. Prevalence of and risk factors for elder abuse and neglect in the community: A population-based study. J Am Geriatr Soc 2015;63(9):1906-1912. https://doi. org $/ 10.1111 /$ jgs. 13601

10. Baker P, Francis D, Hairi N, et al. Interventions for preventing abuse in the elderly. Cochrane Database Syst Rev 2016, Issue 8. Art. No.: CD010321. https://doi.org/10.1002/14651858.cd010321.pub2

Accepted 29 July 2019. 